Cite this: Phys. Chem. Chem. Phys., 2012, 14, 2056-2064

\title{
Limonene: electronic state spectroscopy by high-resolution vacuum ultraviolet photoabsorption, electron scattering, He(I) photoelectron spectroscopy and $a b$ initio calculations $\dagger$
}

\author{
M. A. Śmiałek, ${ }^{* a}$ M.-J. Hubin-Franskin, ${ }^{b}$ J. Delwiche, ${ }^{b}$ D. Duflot, ${ }^{c}$ N. J. Mason, ${ }^{d}$ \\ S. Vronning-Hoffmann, ${ }^{e}$ G. G. B. de Souza, ${ }^{f}$ A. M. Ferreira Rodrigues,${ }^{f}$ \\ F. N. Rodrigues ${ }^{f}$ and P. Limão-Vieira*g \\ Received 7th September 2011, Accepted 1st December 2011 \\ DOI: $10.1039 / \mathrm{c} 2 \mathrm{cp22847e}$
}

Electronic state spectroscopy of limonene has been investigated using vacuum ultraviolet photoabsorption spectroscopy in the energy range $5.0-10.8 \mathrm{eV}$. The availability of a high resolution photon beam $(\sim 0.075 \mathrm{~nm})$ enabled detailed analysis of the vibrational progressions and allowed us to propose, for the first time, new assignments for several Rydberg series. Excited states located in the 7.5-8.4 eV region have been studied for the first time. A He(I) photoelectron spectrum has also been recorded from 8.2 to $9.5 \mathrm{eV}$ and compared to previous low resolution works. A new value of $8.521 \pm 0.002 \mathrm{eV}$ for the ground ionic state adiabatic ionisation energy is proposed. Absolute photoabsorption cross sections were derived in the $10-26 \mathrm{eV}$ range from electron scattering data. All spectra presented in this paper represent the highest resolution data yet reported for limonene. These experiments are complemented by new ab initio calculations performed for the three most abundant conformational isomers of limonene, which we then used in the assignment of the spectral bands.

\section{Introduction}

Monoterpenes have been used for centuries as main components of fragrant oils in perfumes and cosmetics. They can be extracted from leaves, flowers and fruits and exhibit various interesting properties like antimicrobial activity or inhibition of seed germination. ${ }^{1}$ Monocyclic terpenes are derived from cyclohexane and usually possess an isopropyl group. Often they exist in various enantiomeric forms that differ in organoleptic properties.

${ }^{a}$ Atomic Physics Division, Department of Atomic Physics and Luminescence, Faculty of Applied Physics and Mathematics, Gdańsk University of Technology, Gabriela Narutowicza 11/12, 80-952 Gdańsk, Poland. E-mail: smialek@mif.pg.gda.pl;

Fax: + 48 583471705; Tel: + 48583471936

${ }^{b}$ Département de Chimie, Université de Liège, Institut de Chimie-Bât.

B6C, B-4000 Liège, Belgium

${ }^{c}$ Laboratoire de Physique des Lasers, Atomes et Molécules (PhLAM), UMR CNRS 8523, Université Lille1 Sciences et Technologies, F-59655 Villeneuve d' Ascq Cedex, France

${ }^{d}$ Department of Physics and Astronomy, The Open University, Walton Hall, Milton Keynes, MK7 6AA, UK

${ }^{e}$ Institute for Storage Ring Facilities, Aarhus University, Ny Munkegade, Building 1520, DK-8000 Aarhus C, Denmark

${ }^{f}$ Instituto de Quimica, Universidade Federal do Rio de Janeiro,

Ilha do Fundão, 21949-900, Rio de Janeiro, RJ, Brazil

${ }^{g}$ Department of Physics, Universidade Nova de Lisboa,

2829-516 Caparica, Portugal.E-mail: plimaovieira@fct.unl.pt;

Fax: + 35 1212948549; Tel: + 351212947859

$\dagger$ Electronic supplementary information (ESI) available. See DOI: $10.1039 / \mathrm{c} 2 \mathrm{cp} 22847 \mathrm{e}$
One of the most common cyclic monoterpenes is limonene, an abundant component of essential oils extracted from lemon and orange rinds in $R-(+)$ form. The $S-(-)$ enantiomer also occurs naturally in the form of volatiles emitted by oaks and pines. $^{2}$ Due to its orange odour, limonene is widely used in food and cosmetic industry. It was also shown that limonene together with other terpenoids can be a very effective anticancer agent. ${ }^{3}$ In addition, limonene is a major precursor to another flavouring compound, carvone.

The first study of excited states of limonene was performed using photoabsorption, photoionisation and CD spectroscopy techniques in the VUV range of the light spectrum. ${ }^{4}$ The value for the first valence transition, localised on a diene chromophore, was found to be $6.525 \mathrm{eV}$, whereas the first Rydberg transition for the lowest ionisation energy, $8.54 \mathrm{eV}, \pi \rightarrow 3 \mathrm{~s}$, was identified at $5.701 \mathrm{eV}$. Ionisation energies of higher accuracy than the ones derived previously ${ }^{4}$ were obtained together with the most probable ionisation pathways in the threshold region of photoionisation. Photoionisation and parent ion fragmentation of limonene was later investigated in more detail using synchrotron light up to $11 \mathrm{eV}^{5}$ and supported by OVGF calculations.

Ab initio calcutations have shown that limonene possesses six conformational isomers, out of which three are abundant in gaseous samples. ${ }^{6}$ These have been detected using IR, Raman and VCD techniques and all 72 vibrational modes have been calculated and experimentally assigned. ${ }^{7}$ 
In the present work we provide a more detailed electronic states analysis for $R-(+)$-limonene through high resolution VUV photoabsorption and photoelectron spectroscopies combined with ab initio calculations.

\section{Structure and properties of limonene}

In the ground state limonene belongs to a $C_{1}$ symmetry point group and can be found in $R-(+)$ and $S-(-)$ isomeric forms as well as a racemic mixture containing both isomers. The $R$ - $(+)$-limonene molecule, which is an object of this work, has 72 vibrational normal modes in its ground state and belongs to an (A) representation, which is irreducible.

According to previous work there are six possible stable conformers, of which three will have significant abundances at room temperature ${ }^{6}$ in the gas phase, designated as down-I, down-II and down-III. Their geometries have been optimised at the MP2/cc-pVDZ level showing main differences in the value of the torsion angle of the isopropenyl group with respect to the ring (Fig. 1). The energies were then calculated at the CCSD level using the MP2 geometries and their relative values are shown in Table 1. The inclusion of zero-point vibrational energy, when compared to values shown previously, ${ }^{6}$ does not seem to have great impact. The values are almost identical because the three isomers have very similar zero-point energies.

This molecule has 76 electrons, therefore the electronic configuration of the ${ }^{1} A$ ground state contains 38 doubly occupied MOs, all of symmetry a. The 10 lowest lying MOs correspond to the $1 \mathrm{~s}$ carbon orbitals. The highest occupied MO (HOMO, $38 \mathrm{a}, 2 \pi$ ) corresponds to the $\pi$ bond in the carbon ring, while the second highest occupied MO (SHOMO, 37a, $1 \pi$ ) is the $\pi$ bond of the isopropenyl group $\mathrm{C}=\mathrm{CH}_{2} \mathrm{CH}_{3}$ (Fig. 2). The LUMO $\left(1 \pi^{*}\right)$ is the anti-bonding counterpart of the SHOMO. Thus, the

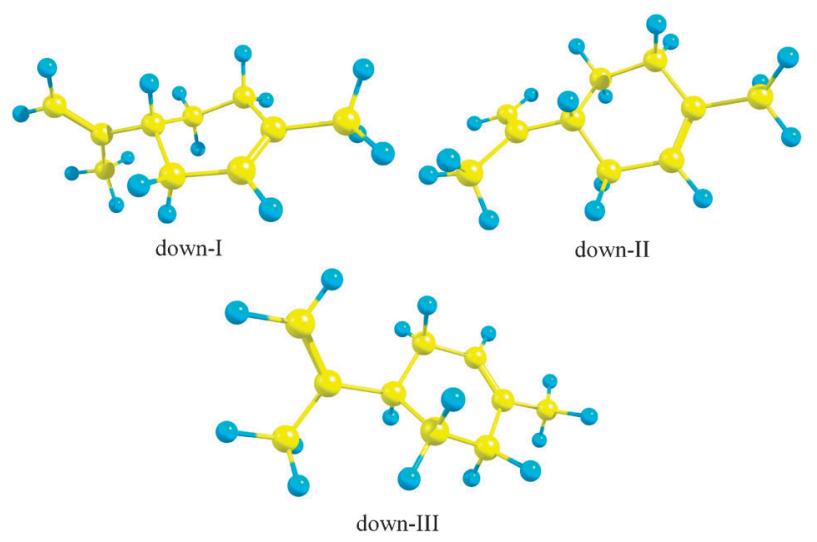

Fig. 1 Three most abundant isomers of limonene.

Table 1 Relative energies of three most abundant isomers of limonene calculated without (MP2) and with (CCSD) inclusion of zero-point vibrational energies and their Boltzmann factors

\begin{tabular}{lllll}
\hline & $\begin{array}{l}\Delta E \mathrm{MP} 2 / \\
\mathrm{kcal} \mathrm{mol}\end{array}$ & $\begin{array}{l}\Delta E+\mathrm{ZPE} / \\
\mathrm{kcal} \mathrm{mol}\end{array}$ & \% (Boltzmann) & $\begin{array}{l}\Delta E \mathrm{CCSD} / \\
\mathrm{kcal} \mathrm{mol}\end{array}$ \\
\hline Down-I & 0.00 & 0.00 & 54.65 & 0.00 \\
Down-II & 0.34 & 0.37 & 29.43 & 0.27 \\
Down-III & 0.75 & 0.71 & 15.91 & 0.67 \\
\hline
\end{tabular}
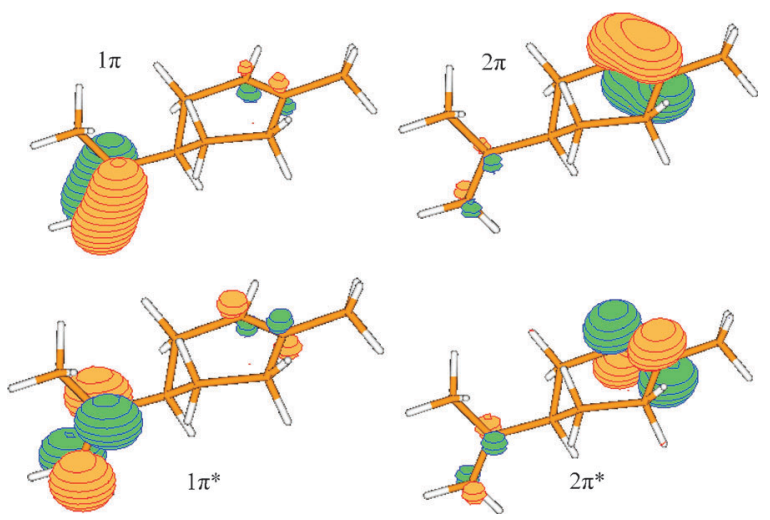

Fig. 2 Localisation of HOMO bonding and LUMO antibonding orbitals of the limonene molecule.

$2 \pi^{*}$ is the anti-bonding MO located on the ring (Fig. 2). A graphical representation of HOMO and LUMO, shown in Fig. 2, was created using the MOLDEN software. ${ }^{8}$ The Cartesian coordinates of atoms for calculated geometries (both the neutral molecule and the ion) of down-isomers are available as ESI. $\dagger$

\section{Experimental}

\subsection{Limonene sample}

The liquid sample used both in the VUV measurements and the PES experiment was purchased from Fluka, with a purity of $99 \%$. The sample was degassed by a repeated freeze-pumpthaw cycle in both sets of experiments.

\subsection{VUV photoabsorption}

High-resolution VUV photoabsorption spectra of limonene were measured at the UV1 beam line using the ASTRID synchrotron facility in Aarhus University, Denmark (Fig. 3). The experimental apparatus has been described in detail previously. ${ }^{9}$ Synchrotron radiation passes through a static gas sample and a photomultiplier is used to measure the transmitted light intensity. The incident wavelength is selected using a toroidal dispersion grating with 2000 lines $\mathrm{mm}^{-1}$ providing a resolution of $\sim 0.075 \mathrm{~nm}$. In order to prevent

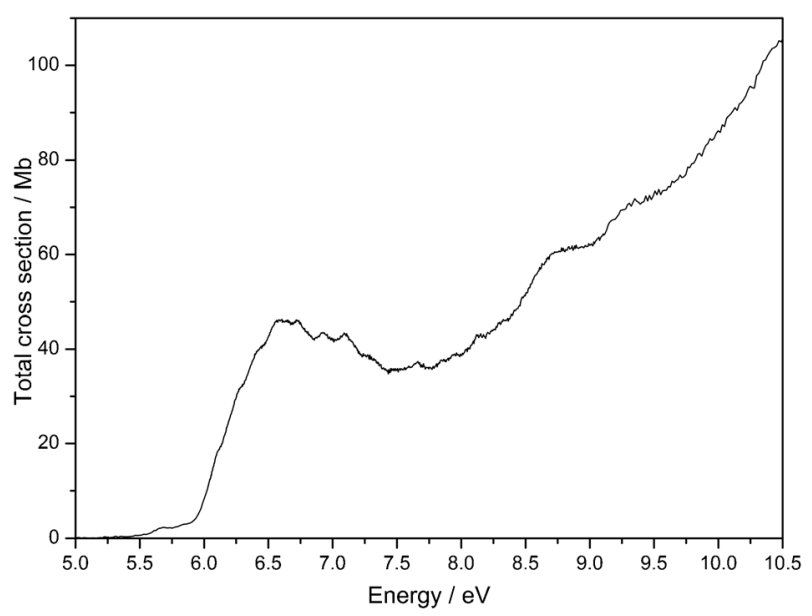

Fig. 3 High resolution VUV photoabsorption spectrum of limonene. 
any absorption by air contributing to the spectrum and for wavelengths below $200 \mathrm{~nm}$, a $\mathrm{He}$ gas flow is maintained through the small gap between the photomultiplier and the exit window of the gas cell. The minimum and maximum wavelengths between which scans are performed, 115-320 nm (10.8-3.9 eV), are determined by the windows of the gas cell (LiF entrance and $\mathrm{MgF}_{2}$ exit) and the grating respectively. The sample pressure is measured using a capacitance manometer (Baratron). To ensure that the data were free of any saturation effects, ${ }^{10,11}$ the cross-section was measured over the pressure range $0.008-0.038$ Torr with typical attenuations of less than $10 \%$. The synchrotron beam ring current is monitored throughout the collection of each spectrum and a background scan recorded with the cell evacuated. Absolute photoabsorption cross sections are then obtained using the Beer-Lambert attenuation law

$$
I_{\mathrm{t}}=I_{0} \times \exp (-n \sigma x),
$$

where $I_{\mathrm{t}}$ is the radiation intensity transmitted through the gas sample, $I_{0}$ is that through the evacuated cell, $n$ is the molecular number density of the sample gas, $\sigma$ is the absolute photoabsorption cross section, and $x$ is the absorption path length $(25 \mathrm{~cm})$. The accuracy of the cross section is estimated to be better than $\pm 5 \%$.

\subsection{Electron scattering}

While in photoabsorption experiments quantitative cross section measurements are governed by the Beer-Lambert law, in electron impact methods the essential parameter is the generalized oscillator strength $f_{n}(K)$, which is related to the differential inelastic electron impact cross section $\left[\partial^{2} \sigma / \partial E \partial \Omega\right]$ by the equation

$$
f_{n}(K)=(E / 2)\left(k_{0} / k_{n}\right) K^{2}\left[\partial^{2} \sigma / \partial E \partial \Omega\right],
$$

where $E$ is the electron energy loss, $k_{0}$ and $k_{n}$ are the incident and scattered electron momenta, $K$ is the momentum transfer and $\Omega$ is the solid scattering angle. In practice, measured oscillator strengths are differential (i.e. $\mathrm{d} f / \mathrm{d} E$ ) in the discrete as well as in the continuum regions. In the first case this is due to the finite linewidth and spectrometer bandwidth. In the continuum region this is due to the continuous nature of the final states. Under experimental conditions in which the momentum transfer is negligible (high incident electron energy, near zero degree scattering angle), dipole selection rules apply and the generalized oscillator strength becomes identical to the optical oscillator strength, $f_{n}^{0}(E)$. By the same reasoning the differential generalized oscillator strength becomes identical to the differential optical oscillator strength. ${ }^{12}$ In the present work the determination of absolute photoabsorption cross sections for the limonene molecule was extended to a much larger energy range using the electron impact technique.

First, the relative intensity of the generalized oscillator strength was measured using an intermediate energy, variableangle, low resolution spectrometer which has been described before. ${ }^{13,14}$ Briefly, the spectrometer consists of an electron gun, a gas inlet system at $90^{\circ}$ with respect to the electron beam, a Mollenstedt electron analyser and a conventional detection system. The electron gun, set at $1 \mathrm{keV}$, can be rotated from $-60^{\circ}$ to $+60^{\circ}$. A $2.0^{\circ}$ energy-loss spectrum was obtained for the limonene molecule in the 4 to $50 \mathrm{eV}$ energy-loss range. The energy resolution was $0.8 \mathrm{eV}$. The limonene spectrum was converted into relative differential generalized strength and then extrapolated to the optical limit using the universal formula of Msezane and Sakmar. ${ }^{15}$ The extrapolation procedure has been discussed in detail elsewhere. ${ }^{16}$

From the relative optical oscillator strength (or more appropriately the differential optical oscillator strength, $\left.\mathrm{d} f_{n}^{0}(E) / \mathrm{d} E\right)$, the corresponding photoabsorption cross section may be obtained through the equation ${ }^{17}$

$$
\sigma[\text { Mbarn }]=109.76 \times \mathrm{d} f_{n}^{0}(E) / \mathrm{d} E\left[\mathrm{eV}^{-1}\right]
$$

The cross section values were afterwards made absolute by normalization at $9.0 \mathrm{eV}$ to the absolute photoabsorption cross section previously obtained with synchrotron radiation, in the 5.0 to $10.5 \mathrm{eV}$ photon energy range.

\subsection{Photoelectron spectroscopy}

$\mathrm{He}(\mathrm{I})(21.22 \mathrm{eV})$ photoelectron spectra of limonene were recorded at the Universite de Liège, Belgium (Fig. 4). The experimental apparatus has been described in detail elsewhere. ${ }^{18}$ The spectrometer consists of a $180^{\circ}$ cylindrical electrostatic analyser with a mean radius of $5 \mathrm{~cm}$. The analyser is used in constant pass energy mode and is fitted with a continuous dynode electron multiplier. Incident photons are produced by a dc discharge in a two stage differentially pumped lamp. The energy scale is calibrated in a standard manner ${ }^{19}$ using the ${ }^{2} \mathrm{P}_{\frac{3}{2}}$ and ${ }^{2} \mathrm{P}_{\frac{1}{2}}$ peaks of $\mathrm{Ar}^{+}$and the spectra are corrected for the transmission function of the apparatus. The resolution of the present spectrum is measured from the FWHM of the $\mathrm{Ar}^{+}$peaks to be $24 \mathrm{meV}$, in the presence of limonene. The accuracy of the energy scale is estimated to be $\pm 2 \mathrm{meV}$. The photoelectron spectrum presented in this paper is the sum of 323 individual spectra. This procedure allows us to obtain a good signal-to-noise ratio while keeping the pressure in the spectrometer at a very low level $\left(<2 \times 10^{-6}\right.$ mbar $)$.

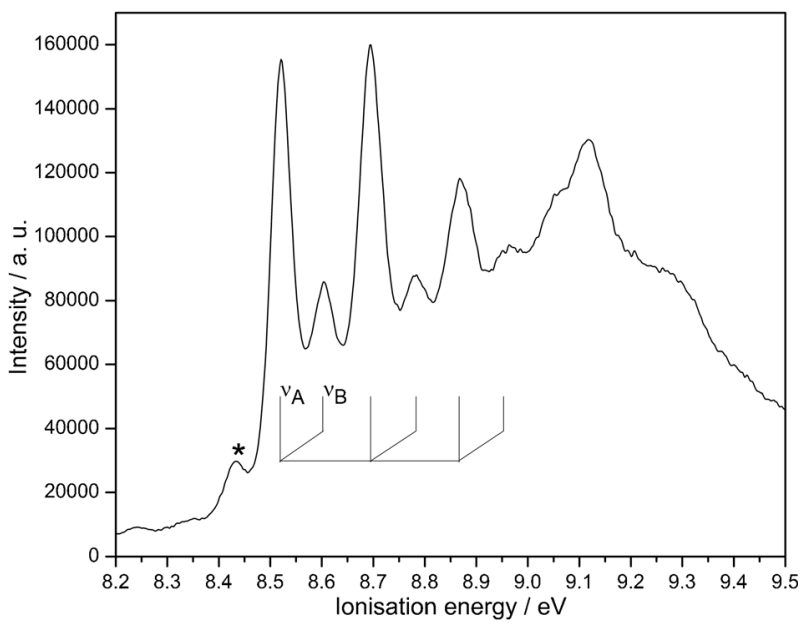

Fig. $4 \mathrm{He}(\mathrm{I})$ photoelectron spectrum of limonene, $\mathrm{C}_{10} \mathrm{H}_{16}$, with vibronic excitation assignment; asterisk marks a feature that can correspond to a dimer presence. 


\section{Computational methods}

$A b$ initio calculations were performed using the MOLPRO programme..$^{20}$ First, the ground state geometry of the three isomers was optimised at the MP2 level using the Dunnings cc-pVDZ basis set. ${ }^{21}$ Harmonic vibrational frequencies were also computed at MP2 level to confirm the nature (minima or saddle points) of the optimised structures. The electronic excitation spectra were calculated at these geometries using the EOM-CCSD (equation-of-motion coupled cluster method restricted to single and double excitations) level. ${ }^{22}$ Two sets of calculations were performed. The first one used the cc-pVDZ basis, providing pure valence transitions. For the second one, a set of diffuse functions ( $4 \mathrm{~s}, 4 \mathrm{p}, 2 \mathrm{~d}$ ), taken from Kaufmann et $a l .{ }^{23}$ and localized at the centre of the ring, was added to the original basis set in order to obtain Rydberg transitions (cc-pVDZ $+\mathrm{R}$ basis set). The corresponding oscillator strengths were calculated in the length gauge. The lowest ionisation energies of limonene were also obtained at the RCCSD(T)/cc-pVDZ level. ${ }^{24}$ In order to obtain higher ionisation energies, additional partial third order (P3) propagation ${ }^{25,26}$ calculations were performed using the Gaussian09 package. ${ }^{27}$

Concerning the geometry of ionised limonene, it is logical to employ the same level of computation as that for the neutral molecule, i.e., MP2. However, it is well $\mathrm{known}^{28}$ that in the case of open shell systems, there are several possible MP2 methods. The method we have chosen in the present work is the ZAPT technique, ${ }^{29}$ implemented within the GAMESS-US program. ${ }^{30,31}$ Consequently, harmonic frequencies for the ions were obtained numerically rather than analytically. Finally, in order to interpret the vibrational structure we used the ezSpectrum program. ${ }^{32,33}$ These calculations take into account the Duschinsky rotations between the ground and the ion vibrations ${ }^{34}$ for the 72 vibrational modes of the molecule, and include hot and combination bands.

\section{Results and discussion}

The total cross-section for VUV photoabsorption of limonene is shown in Fig. 3, ranging from 5.0 to $10.8 \mathrm{eV}$. The present spectra are of much higher resolution than those in previously published data ${ }^{4}$ and therefore the fine structure in the $6.5 \mathrm{eV}$ region is better resolved. In addition, for the first time the spectrum was measured above $8.25 \mathrm{eV}$, extending over the ionisation region. The observed bands were assigned to both Rydberg series and molecular valence transitions. In the lowenergy range, from 6.0 to $7.5 \mathrm{eV}$, we suggest pure Rydberg character, whereas in the 7.5 to $8.5 \mathrm{eV}$ region pure valence transitions are suggested.

The photoelecton spectrum shown in this paper was measured with a resolution comparable to the one used previously ${ }^{5}$ and was deconvoluted revealing for the first time additional fine structure. A new value for the adiabatic ionisation energy is suggested to be $8.521 \pm 0.002 \mathrm{eV}$. The values proposed here for vertical ionisation energies are in very good agreement with the theoretical calculations presented both in this work and previously. ${ }^{5}$

The results of theoretical calculations obtained for all three conformers are presented in Tables 3 and 4 and are compared with experimental results. The data obtained from experiments are in a good agreement with theoretical predictions.

\subsection{He(I) photoelectron}

Photoelectron spectroscopy can give both adiabatic and vertical ionisation energies. ${ }^{19}$ The photoelectron spectrum of limonene has already been reported by Garcia et $a l .{ }^{5}$ and Brint et $a .^{4}{ }^{4}$ with values for both the adiabatic and the vertical ionisation energy being derived. In the first electronic band the point of maximum intensity was found at $8.695 \mathrm{eV}$ and thus corresponds to the vertical ionisation energy, whereas the intense feature at $8.521 \mathrm{eV}$ is attributed to the adiabatic ionisation energy. The obtained values are in a good agreement with those obtained previously. ${ }^{4,5}$ In the present experiment, we suggest values of $8.521 \pm 0.002 \mathrm{eV}$ and $8.695 \pm$ $0.002 \mathrm{eV}$ for the adiabatic and vertical ionisation energies respectively. The calculated values, shown in the last columns of Table 2, prove that the three isomers have very similar ionisation energies. It should be noticed that it is not sure which conformer was used in Garcias calculations. All the theoretical results predict a slightly lower vertical ionisation energy, close to $8.5 \mathrm{eV}$.

Table 2 Energy positions (in eV) and vibrational analysis of features observed in the first and second photoelectron bands of limonene in this work together with previous results for limonene

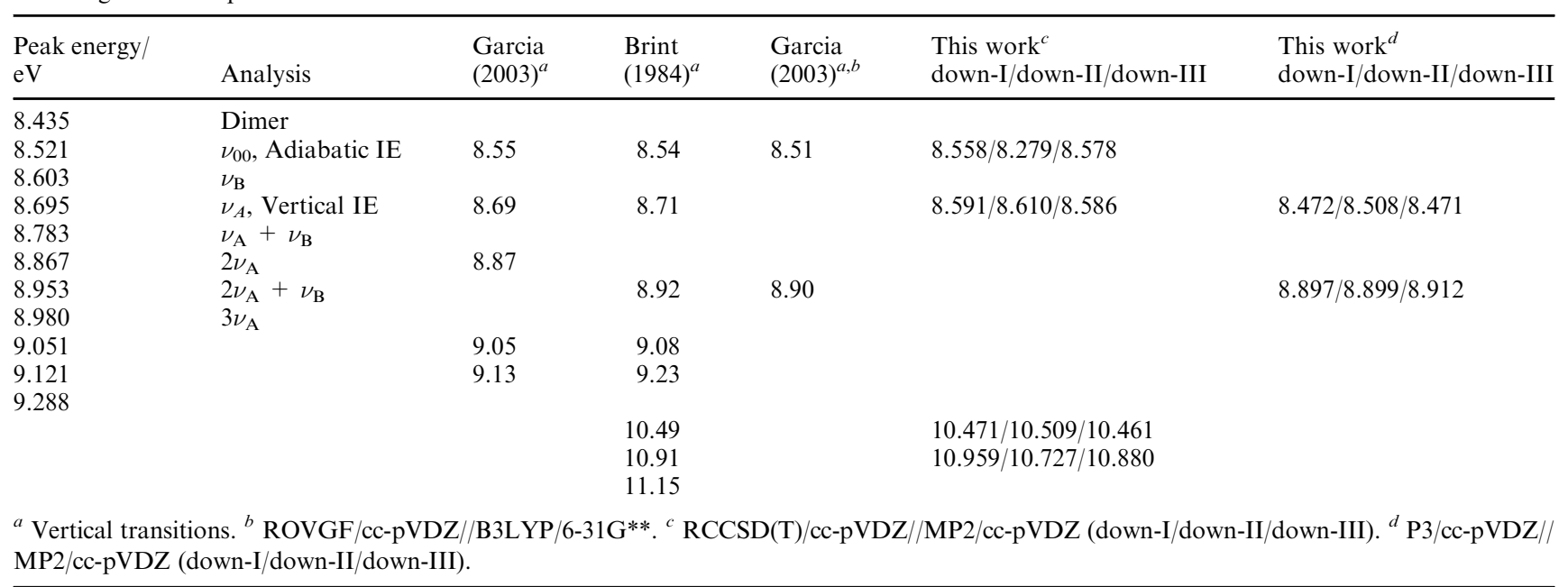


The first band of the spectrum, corresponding to the ionic electronic ground state of limonene, is seen below $9 \mathrm{eV}$ in Fig. 4. The fine structure in this energy band is resolved here for the first time and shows two vibrational progressions with mean energies of 0.172 and $0.086 \mathrm{eV}$ (Table 2), respectively. Another feature is also reported in the low energy part of the spectrum and is tentatively assigned to the presence of a dimer with an energy spacing of $0.086 \mathrm{eV}$. This is marked with an asterisk in Fig. 4.

In order to assign these observations, Franck-Condon factors were calculated at $300 \mathrm{~K}$ for all three isomers of limonene and are shown in Fig. 5. It can be seen that the values of Franck-Condon factors are different for all three isomers, with much larger values for the down-II isomer. Thus, if we make the reasonable assumption that the electronic transition moments from the neutral molecule to the ion are close for the three isomers, we can rely on the down-II spectrum only for interpretation purposes. A close examination of the most intense vibrational bands reveals that the observed $0.172 \mathrm{eV}$ strong progression corresponds to the $\nu_{55}$ mode,
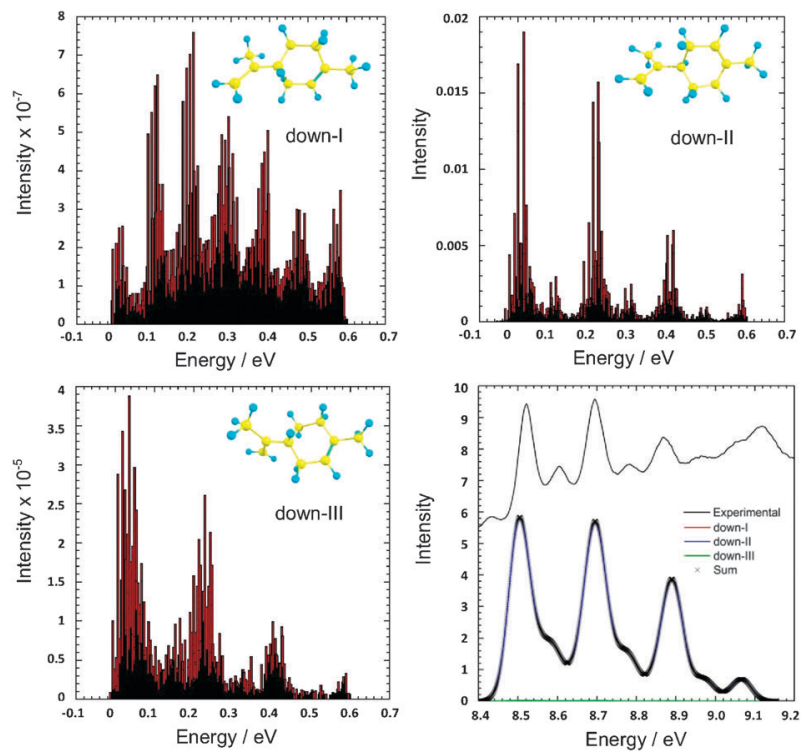

Fig. 5 The Franck-Condon factors calculated for down-I, down-II and down-III isomers together with their superposition weighted by Boltzmann factors (the black solid line is the actual photoelectron spectrum); the geometries shown as insets were calculated at the ZAPT/cc-pVDZ level. calculated at $0.197 \mathrm{mV}$. This mode is essentially the stretching of the ring $\mathrm{C}=\mathrm{C}$ double bond. The $0.086 \mathrm{eV}$ observed progression may be assigned to two similar modes $\left(\nu_{17}\right.$ and $\nu_{18}$ ), calculated at 0.086 and $0.089 \mathrm{eV}$. The first one corresponds to the out-of-plane movement of the hydrogen atom connected to the ring $\mathrm{C}=\mathrm{C}$ bond, while the second one is more delocalised and involves several $\mathrm{H}$ atoms around the whole molecule. These results are consistent with the location of the $2 \pi$ HOMO (Fig. 1), which is emptied upon ionization: this $\mathrm{MO}$ is located on the ring $\mathrm{C}=\mathrm{C}$ double bond and it is logical that vibrational excitation should involve the atoms present in this part of the molecule. As can be seen in the ESI $\dagger$, the geometries of the ions are almost identical to their neutral counterparts, the largest difference being for down-I, which presents a torsion of the isopropenyl group. The weighted root-mean-square distances between the neutral molecule and the ion are typically twice as large for down-I and down-III ( $0.30 \AA$ and $0.36 \AA$, respectively), than for down-II $(0.15 \AA)$. This explains the low FC factors and the more important vibrational excitation, as can been seen in Fig. 5, for down-I and down-III isomers. Finally, the low energy band assigned to the hot band does not appear in the simulated spectra, even if the temperature is raised to $900 \mathrm{~K}$. They are present in the calculations, but are too weak to be visible. Moreover, they involve the lowest lying frequencies (typically a few $\mathrm{meV}$, methyl groups rotation modes), and cannot explain the feature observed at $0.086 \mathrm{eV}$ below the $0-0$ peak. As displayed in the last part of Fig. 5, except for this hot band and the contribution of the second state, the calculated spectrum (FWHM = $0.05 \mathrm{eV}$ ) is very similar to the observed one.

Concerning the second electronic band, all calculations agree to predict only one excited electronic state below $10 \mathrm{eV}$ (Table 2). The vertical excitation for the second electronic state was calculated to be $8.90 \mathrm{eV}$ by Garcia et al. ${ }^{5}$ Our own P3 results (last column of Table 2) confirm this result and prove that the vertical ionisation energy is around $8.9 \mathrm{eV}$ for the three conformers.

\subsection{The Rydberg series}

The photoabsorption spectrum shows a large structure centred around $6.5 \mathrm{eV}$. Computation revealed that it originates mainly from transitions from $2 \pi$ and $1 \pi$ orbitals to the lowest Rydberg states. Ab initio calculations show that all three conformers will contribute to these transitions and the calculated energies for such transitions to the $n=3$ term together with oscillator

Table 3 Energy values, oscillator strengths and quantum defects of possible Rydberg transitions in limonene derived by ab initio calculations and Rydberg formula

\begin{tabular}{|c|c|c|c|c|c|c|c|c|c|}
\hline \multirow[b]{2}{*}{ Assignment } & \multicolumn{3}{|c|}{$\underline{\text { Down-I }}$} & \multicolumn{3}{|c|}{ Down-II } & \multicolumn{3}{|c|}{ Down-III } \\
\hline & $E / \mathrm{eV}$ & $f$ & $\delta$ & $E / \mathrm{eV}$ & $f$ & $\delta$ & $E / \mathrm{eV}$ & $f$ & $\delta$ \\
\hline $2 \pi \rightarrow 3 \mathrm{~s}$ & 6.006 & 0.0027 & 0.67 & 6.013 & 0.0020 & 0.67 & 6.002 & 0.0026 & 0.68 \\
\hline $1 \pi \rightarrow 3 \mathrm{~s}$ & 6.391 & 0.0253 & 0.47 & 6.447 & 0.0118 & 0.44 & 6.435 & 0.0133 & 0.45 \\
\hline $2 \pi \rightarrow 3 p$ & 6.454 & 0.0142 & 0.43 & 6.470 & 0.0206 & 0.42 & 6.439 & 0.0247 & 0.44 \\
\hline $2 \pi \rightarrow 3 p$ & 6.540 & 0.0107 & 0.38 & 6.555 & 0.0044 & 0.37 & 6.538 & 0.0036 & 0.38 \\
\hline $2 \pi \rightarrow 3 p$ & 6.674 & 0.0026 & 0.22 & 6.761 & 0.0033 & 0.22 & 6.755 & 0.0002 & 0.22 \\
\hline $1 \pi \rightarrow 3 p$ & 6.897 & 0.0228 & 0.11 & 6.880 & 0.0591 & 0.12 & 6.888 & 0.0364 & 0.11 \\
\hline $1 \pi \rightarrow 3 p$ & 6.942 & 0.0350 & 0.06 & 7.015 & 0.0129 & -0.01 & 6.995 & 0.0236 & 0.01 \\
\hline $1 \pi \rightarrow 3 p$ & 7.121 & 0.0054 & -0.12 & 7.198 & 0.0017 & -0.18 & 7.176 & 0.0002 & -0.14 \\
\hline $2 \pi \rightarrow 3 d$ & 7.144 & 0.0129 & -0.14 & 7.175 & 0.0260 & -0.21 & 7.145 & 0.0159 & -0.18 \\
\hline
\end{tabular}




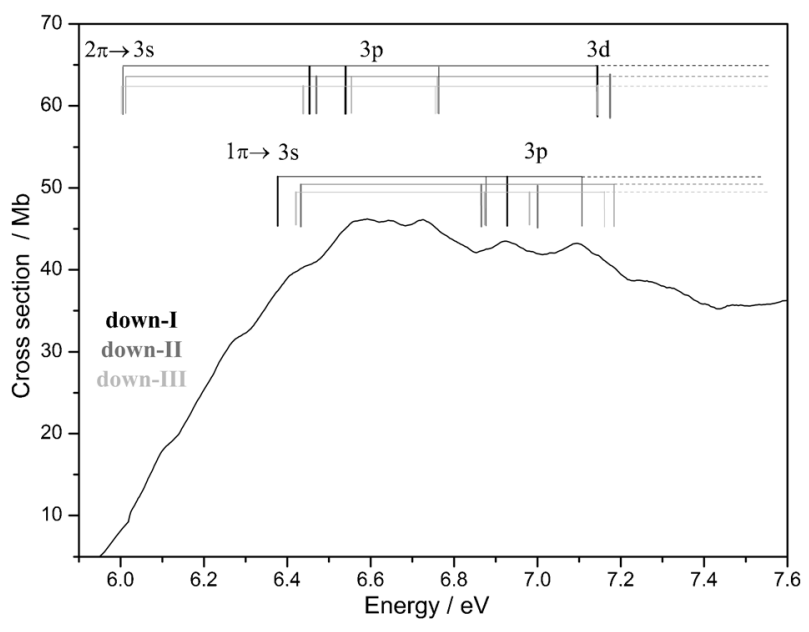

Fig. 6 Rydberg series assignment for $2 \pi$ and $1 \pi$ progressions computed for three conformers.

strengths are shown in Table 3. Because all three isomers are present in the sample and oscillator strength values are similar for these conformers, it was difficult to clearly match calculated transitions to the features present in the spectrum.

Calculated values are additionally plotted in Fig. 6, where the thick lines in the Rydberg series mark the transitions of oscillator strengths greater than 0.01 . On that basis, using the Ryberg formula $E_{n}=E_{\mathrm{i}}-R /(n-\delta)^{2}$, where $E_{\mathrm{i}}$ is the ionisation energy (adiabatic value, $E_{\mathrm{i}}=8.521 \mathrm{eV}$ ), $n$ is the principal quantum number of the Rydberg orbital of energy $E_{n}, R$ is one Rydberg and $\delta$ is the quantum defect, it was possible to obtain the values for quantum defects in limonene. The values obtained were $0.7,0.45-0.25$ and $\sim 0$ for $n \mathrm{~s}, n \mathrm{p}$ and $n \mathrm{~d}$ transitions from the $2 \pi$ orbital and 0.45 and $0.12-0$ for $n \mathrm{~s}$ and $n \mathrm{p}$ transitions from the $1 \pi$ orbital. The values of Rydberg series were then calculated for $n=3$ to 11 and plotted for transitions from the $2 \pi$ state, Fig. 7 , and from the $1 \pi$ state, Fig. 8, for the most abundant topoisomer of limonene, down-I.

It was also possible to assign some vibrational excitation modes in the spectrum (Fig. 9). Four modes were assigned, labeled as $\nu_{\mathrm{A}}=172 \mathrm{meV}, \nu_{\mathrm{B}}=86 \mathrm{meV}, \nu_{\mathrm{C}}=206 \mathrm{meV}$ and $\nu_{\mathrm{D}}=351 \mathrm{meV}$. According to analysis shown previously, ${ }^{7}$ it was possible to assign $\nu_{\mathrm{A}}$ to mode $\nu_{26}$, which corresponds to

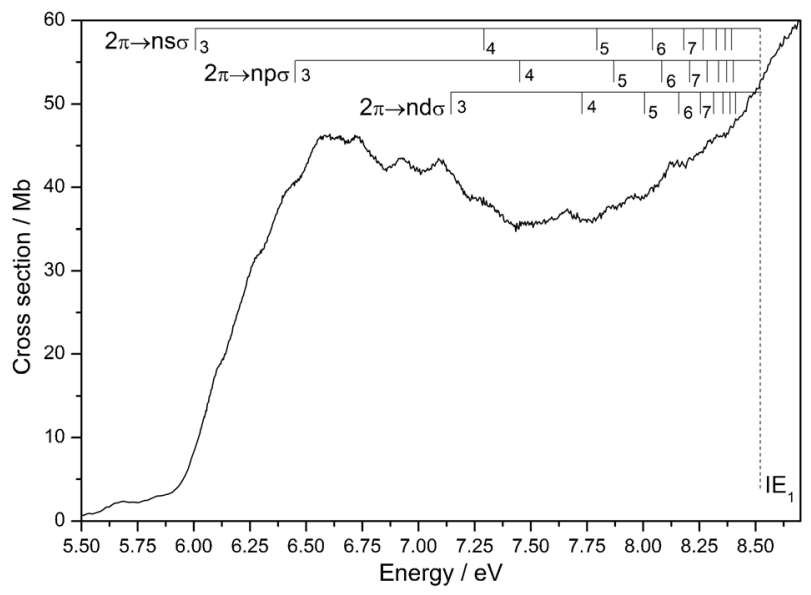

Fig. 7 Assignment of $n \mathrm{~s}, n \mathrm{p}$ and $n$ d Rydberg series from $2 \pi$ HOMO.

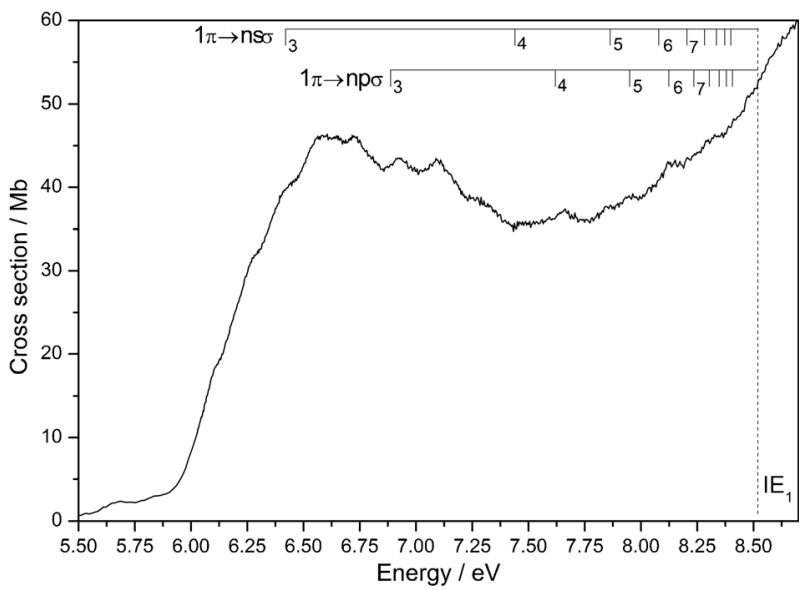

Fig. 8 Assignment of $n$ s and $n$ p Rydberg series from $1 \pi$ SHOMO.

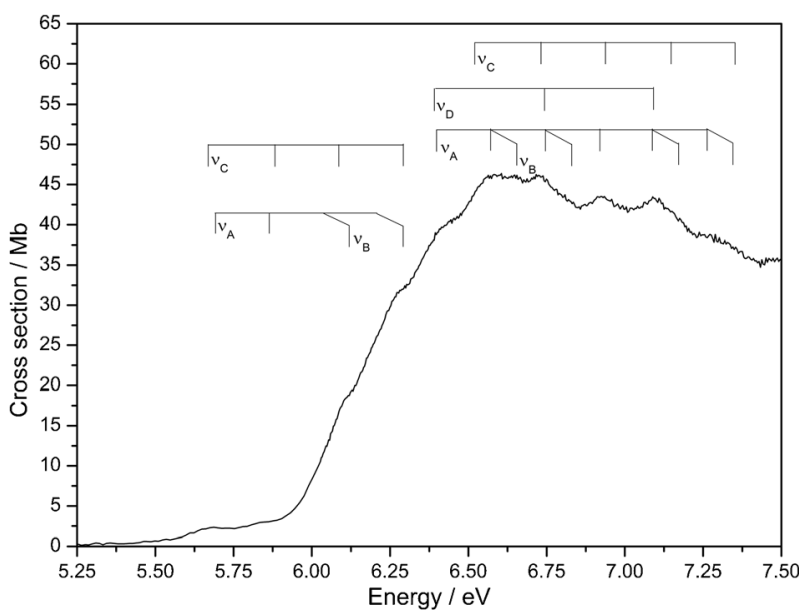

Fig. 9 Vibrational excitation assignment in the low energy region.

$\mathrm{C}-\mathrm{H}$ bending coupled with in-plane bending of the terminal methylene group on the vinyl site and $\nu_{\mathrm{B}}$ to $\nu_{56}$ that is $\mathrm{C}-\mathrm{H}$ group deformation (rocking). The $\nu_{\mathrm{C}}$ mode could be assigned to $\nu_{17-18}$, being either the $\mathrm{C}=\mathrm{C}$ stretching of the vinyl group mixed with the symmetric deformation of the $\mathrm{H}-\mathrm{C}-\mathrm{H}$ angle at the same group $\nu_{17}$ or the $\mathrm{C}=\mathrm{C}$ stretching on the ring $\nu_{18}$. Mode $\nu_{\mathrm{D}}$ was matched with the $\nu_{16}$ mode, corresponding to combination of $\mathrm{C}-\mathrm{H}$ vibrations of the methylene group and symmetric stretching on the vinyl group.

\subsection{Valence states and transitions of limonene}

Computations predict that the region from 7.5 to $8.5 \mathrm{eV}$ in the VUV photoabsorption spectrum will contain pure valence transitions. This region is shown in Fig. 10. The calculated values for the energies and oscillator strengths in this region for the three conformers are shown in Table 4.

Due to the large increase in cross section, it was difficult to identify all the possible valence transitions as well as possible underlying dissociative states predicted by the calculations. We propose to assign the structure centred at $7.658 \mathrm{eV}$ to the $2 \pi \rightarrow 2 \pi^{*}$ transition, whereas the one peaking at $8.042 \mathrm{eV}$ may be assigned to $1 \pi \rightarrow 1 \pi^{*}$ transition.

Vibrational excitations were also found in this part of the spectrum and are shown in Fig. 10. All four modes, described 


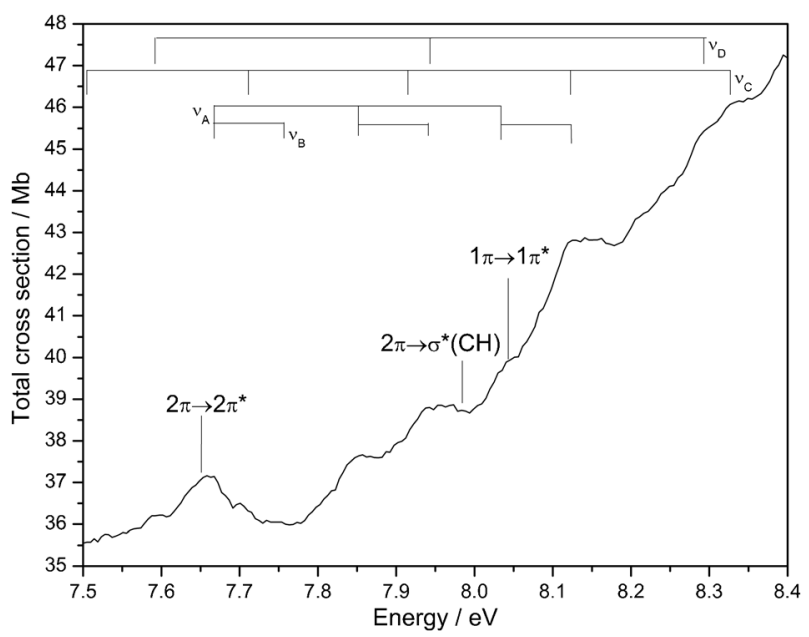

Fig. 10 Valence transitions and vibrational progressions in $7.5-8.5 \mathrm{eV}$ region.

Table 4 Energy values and oscillator strengths calculations of possible pure valence transitions in limonene

\begin{tabular}{|c|c|c|c|c|c|c|}
\hline \multirow[b]{2}{*}{ Assignment } & \multicolumn{2}{|c|}{ Down-I } & \multicolumn{2}{|c|}{ Down-II } & \multicolumn{2}{|c|}{ Down-III } \\
\hline & $E / \mathrm{eV}$ & $f$ & $E / \mathrm{eV}$ & $f$ & $E / \mathrm{eV}$ & $f$ \\
\hline $2 \pi \rightarrow 2 \pi^{*}$ & 7.635 & 0.3929 & 7.667 & 0.3486 & 7.636 & 0.2399 \\
\hline $2 \pi \rightarrow \sigma^{*}(\mathrm{CH})$ & 8.000 & 0.1914 & 7.983 & 0.0980 & 7.983 & 0.1057 \\
\hline $1 \pi \rightarrow 1 \pi^{*}$ & 8.029 & 0.2846 & 8.091 & 0.3816 & 8.018 & 0.3658 \\
\hline $1 \pi \rightarrow \sigma^{*}(\mathrm{CH})$ & 8.228 & 0.0189 & 8.341 & 0.0130 & 8.259 & 0.0140 \\
\hline $1 \pi / \sigma(\mathrm{CC}) \rightarrow 2 \pi^{*} / \sigma^{*}(\mathrm{CH})$ & 8.438 & 0.0177 & 8.416 & 0.0095 & 8.379 & 0.1336 \\
\hline $2 \pi / \sigma(\mathrm{CC}) \rightarrow 1 \pi^{*}$ & 8.464 & 0.0239 & 8.470 & 0.0025 & 8.482 & 0.0075 \\
\hline $2 \pi / \sigma(\mathrm{CH}) \rightarrow 1 \pi^{*}$ & 8.641 & 0.0015 & 8.613 & 0.0066 & 8.698 & 0.0085 \\
\hline $2 \pi / \sigma(\mathrm{CH}) \rightarrow 1 \pi^{*}$ & 8.769 & 0.0108 & 8.683 & 0.0060 & 8.771 & 0.0186 \\
\hline$\sigma(\mathrm{CC}) \rightarrow 2 \pi^{*}$ & 8.878 & 0.0040 & 8.919 & 0.0011 & 8.880 & 0.0168 \\
\hline
\end{tabular}

in Section 5.2 were found to be excited in the higher energy region, which is consistent with the vibrational modes observed in the lowest ionic band of limonene.

\subsection{Extended energy-range (4.0-50.0 eV) absolute photoabsorption cross sections and static dipole polarizability for the limonene molecule}

The oscillator strength distribution for the limonene molecule, determined through electron energy-loss spectroscopy in the 4-50 eV energy range, was converted to photoabsorption cross section using the procedure described in Section 3.3. The results were normalized to the absolute photoabsorption cross section (Fig. 3) at $9.0 \mathrm{eV}$. The complete absolute photoabsorption cross section, encompassing the 4.0 to $50.0 \mathrm{eV}$ energy range, is presented in Fig. 11. The error bars are approximately $5 \%$ in the optical case and $10 \%$ in the electron impact method.

From the absolute values for the differential oscillator strength $(\mathrm{d} f / \mathrm{d} E)$ distribution the static dipole polarizability, $\alpha$, for the limonene molecule may be obtained, by applying the $\mathrm{S}(-2)$ sum rule ${ }^{12,35}$

$$
\mathrm{S}(-2)=\int\left(E / E_{\mathrm{H}}\right)^{-2}(\mathrm{~d} f / \mathrm{d} E) \mathrm{d} E=\alpha,
$$

where $E_{\mathrm{H}}$ is the Hartree energy constant $(27.21 \mathrm{eV})$. In the sum rule normalization procedure the contribution of the target molecule to the photoabsorption above the upper limit of the

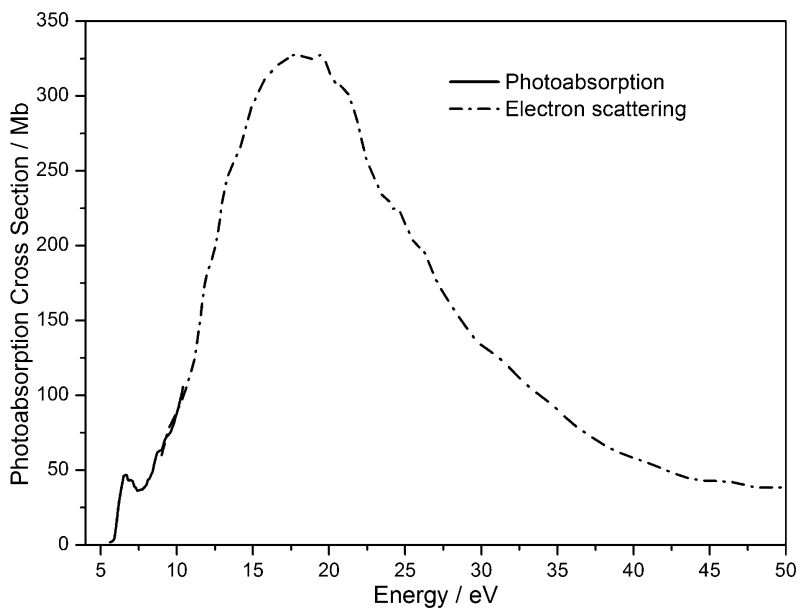

Fig. 11 Extended (4.0-50.0 eV) absolute photoabsorption cross sections for the limonene molecule as derived from photoabsorption (solid line) and electron scattering (dash-dot line) data.

measured spectrum $(50 \mathrm{eV})$ has been estimated by fitting a polynomial of the form

$$
\mathrm{d} f / \mathrm{d} E=A E^{-2}+B E^{-3}+C E^{-4}
$$

to the smooth continuum of the relative photoabsorption data and extrapolating to infinite energy. This sum rule procedure has been successfully applied to a large number of atoms and molecules by the Vancouver group, led by C. E. Brion. Both the $\mathrm{S}(0)$ or Thomas-Reiche-Kuhn sum rule and the $\mathrm{S}(-2)$ sum rule were ingeniously employed by this group in the determination of experimental absolute dipole oscillator strengths for valence-shell and core excitation of atoms and molecules. ${ }^{36}$ Due to the $E^{-2}$ term, the $\mathrm{S}(-2)$ sum rule is heavily weighted in the low energy region and consequently its application does not necessarily require differential oscillator strength measurement up to infinite energy, since data above approximately $50 \mathrm{eV}$ would not make a significant contribution to the $\mathrm{S}(-2)$ sum rule. We obtain a static polarizability value of $\alpha=19.5 \times$ $10^{-24} \mathrm{~cm}^{3}$, in reasonable agreement (within $8.3 \%$ ) with the available polarizability value $\left(17.98 \times 10^{-24} \mathrm{~cm}^{3}\right)$ for the limonene molecule (Chem Guide etc.). This adds further credibility to the present absolute, large photon energy range photoabsorption cross sections for the limonene molecule.

\subsection{Absolute photoabsorption cross-sections and atmospheric photolysis}

Previous absolute VUV photoabsorption cross-sections of limonene are only available in the wavelength ranges 150-225 nm (5.511-8.267 eV). Brint et al. ${ }^{4}$ reported a cross section at $187.5 \mathrm{~nm}(6.0 \mathrm{eV})$ and at $202.5 \mathrm{~nm}(6.1 \mathrm{eV})$ of 20.7 and $9.2 \mathrm{Mb}$, respectively, in contrast to the present values of 46.1 and $19.1 \mathrm{Mb}$, respectively. We believe that these differences may be attributed to the resolution of each apparatus; however other factors such as the role of saturation in other data sets may also lead to the observation of lower cross sections by previous authors.

These absolute cross-sections can be used in combination with solar actinic flux ${ }^{37}$ measurements from the literature to 
estimate the photolysis rate of limonene in the atmosphere from an altitude close to the ground to the stratopause at $50 \mathrm{~km}$. Details of the programme are presented in a previous publication. ${ }^{38}$ The quantum yield for dissociation following absorption is assumed to be unity. The reciprocal of the photolysis rate at a given altitude corresponds to the local photolysis lifetime. Photolysis lifetimes of less than 72 sunlit hours were calculated at altitudes above $22 \mathrm{~km}$. This indicates that limonene molecules can be broken up quite efficiently by VUV absorption at these altitudes. At the ground level photolysis lifetimes increase to several sunlit days.

Rate constants for gas-phase reactions of $\mathrm{OH}$ radicals with limonene have been studied and a value of $4.22 \times$ $10^{-11} \mathrm{~cm}^{3}$ molecule $\mathrm{s}^{-1}$ was measured in a temperature range of 298-364 K, such that these molecules may survive further from their emission sources. ${ }^{39}$ Calculated tropospheric lifetimes of limonene reactions with $\mathrm{OH}, \mathrm{O}_{3}$ and $\mathrm{NO}_{3}$ have yielded values of less than $2 \mathrm{~h},{ }^{40}$ meaning that radical reactions must all be considered as the main loss processes for limonene. This may provide a main reactive sink mechanism in the Earth's atmosphere. Therefore, compared with radical reactions, UV photolysis is not expected to play a significant role in the tropospheric removal of these molecules.

\section{Conclusions}

The present work presents the first high resolution VUV photoabsorption spectrum of limonene together with a high resolution photoelectron spectrum and theoretical analysis. The photolysis lifetimes of limonene have also been carefully derived for the Earth's troposphere and stratosphere.

The results shown in this paper are in a good agreement with the previously published data $^{4}$ but theoretical calculations reveal that the $\pi \rightarrow \pi^{*}$ transition has a higher energy than stated previously. The broad feature in the photoabsorption spectrum observed at $6.5 \mathrm{eV}$ was found to be mainly due to Rydberg transitions. This hypothesis is supported by the ab initio calculations, presented in Table 4. Vibrational series have been resolved in the lowest ionic band, involving mainly $\nu_{26}$, which corresponds to $\mathrm{C}-\mathrm{H}$ bending coupled with in-plane bending of the terminal methylene group on the vinyl site and $\nu_{56}$ that is $\mathrm{C}-\mathrm{H}$ group rocking.

The adiabatic ionisation energy was found to be $8.521 \mathrm{eV}$, which is in a very good agreement with the value given previously ${ }^{5}$ and calculated values of $8.558,8.279$ and $8.578 \mathrm{eV}$ for down-I, down-II and down-III conformers, respectively.

\section{Acknowledgements}

MAS would like to thank the COST CM0603 action for supporting her visit to the Universidade Nova de Lisboa, Portugal. PLV acknowledges the visiting fellow position in the Molecular Physics group, Open University, UK, and together with M-J H-F acknowledges the financial support from the Portuguese-Belgian joint collaboration. The Patrimoine of the University of Liège, the Fonds National de la Recherche Scientifique and the Fonds de la Recherche Fondamentale Collective of Belgium have supported this research. PLV and NJM acknowledge the support from the British Council for the Portuguese-English joint collaboration. The authors wish to acknowledge the beam time at the ISA synchrotron at Aarhus University, Denmark, supported by the EU I3 programme ELISA, grant agreement number 226716. We also acknowledge the financial support provided by the European Commission through the Access to Research Infrastructure action of the Improving Human Potential Programme. GGBS, AMFR and FNR gratefully acknowledge the support from FAPERJ and CNPq (Brazil). Some of this work forms part of the EU/ESF COST Actions Electron Controlled Chemical Lithography (ECCL) CM0601, Astrochemistry CM0805 and Nano IBCT MP1002.

\section{References}

1 H. P. Singh, D. R. Batish, S. Kaur, K. Arora and R. K. Kohli, Ann. Bot., 2006, 98, 1261-1269.

2 J. Schween, R. Dlugi, C. Hewitt and P. Foster, Atmos. Environ. 1997, 31, 199-215.

3 W. Russin, J. Hoesly, C. Elson, M. Tanner and M. Gould, Carcinogenesis, 1989, 10, 2161-2164.

4 P. Brint, E. Meshulam and A. Gedanken, Chem. Phys. Lett., 1984, 109, 383-387.

5 G. Garcia, L. Nahon and I. Powis, Int. J. Mass Spectrom., 2003, 225, 261-270.

6 B. Jansik, A. Rizzo, L. Frediani, K. Ruud and S. Coriani, J. Chem. Phys., 2006, 125, 1-9.

7 F. Partal Urena, J. R. Aviles Moreno and J. J. Lopez Gonzalez, Tetrahedron: Asymmetry, 2009, 20, 89-97.

8 G. Schaftenaar and J. Noordik, J. Comput.-Aided Mol. Des., 2000, 14, 123-134.

9 S. Eden, P. Limao-Vieira, S. Hoffmann and N. Mason, Chem. Phys., 2006, 323, 313-333.

10 N. Mason, J. Gingell, J. Davies, H. Zhao, I. Walker and M. Siggel, J. Phys. B: At., Mol. Opt. Phys., 1996, 29, 3075-3089.

11 W. Chan, G. Cooper and C. Brion, Phys. Rev. A, 1991, 44, 186-204.

12 M. Inokuti, Rev. Mod. Phys., 1971, 43, 297-347.

13 G. G. B. de Souza and A. C. de A de Souza, J. Phys. E: Sci. Instrum., 1985, 18, 1037-1039.

14 C. Bielschowsky, C. Lucas, G. de Souza and J. Nogueira, Phys. Rev. A, 1991, 43, 5975-5979.

15 A. Msezane and I. Sakmar, Phys. Rev. A, 1994, 49, 2405-2410.

16 H. M. Boechat-Roberty, C. A. Lucas, M. C. A. Lopes, M. L. M. Rocco and G. G. B. de Souza, J. Phys. B: At., Mol. Opt. Phys., 2009, 42095201 (6pp).

17 J. Berkowitz, Photoabsorption, Photoionization and Photoelectron Spetroscopy, Academic Press, New York, 1979, p. 469.

18 J. Delwiche, P. Natalis, J. Momigny and J. E. Collin, J. Electron Spectrosc. Relat. Phenom., 1972-1973, 1, 219-225.

19 J. H. D. Eland, Photoelectron spectroscopy; an introduction to ultraviolet photoelectron spectroscopy in the gas phase [by] J. H. D. Eland, Butterworths, London, 1974, p. 239.

20 H. J. Werner, P. J. Knowles, R. Lindh, F. R. Manby, M. Schütz, P. Celani, T. Korona, G. Rauhut, R. D. Amos, A. Bernhardsson, A. Berning, D. L. Cooper, M. J. O. Deegan, A. J. Dobbyn, F. Eckert, C. Hampel, G. Hetzer, A. W. Lloyd, S. J. McNicholas, W. Meyer, M. E. Mura, A. Nicklass, P. Palmieri, R. Pitzer, U. Schumann, H. Stoll, A. J. Stone, R. Tarroni and T. Thorsteinsson, MOLPRO, version 2006.1, a package of ab initio programs, 2007.

21 T. H. Dunning, J. Chem. Phys., 1989, 90, 1007-1023.

22 C. Hampel, K. Peterson and H. Werner, Chem. Phys. Lett., 1992, 190, $1-12$.

23 K. Kaufmann, W. Baumeister and M. Jungen, J. Phys. B: At., Mol. Opt. Phys., 1989, 22, 2223-2240.

24 P. Knowles, C. Hampel and H. Werner, J. Chem. Phys., 1993, 99, 5219-5227.

25 A. M. Ferreira, G. Seabra, O. Dolgounitcheva, V. G. Zakrzewski and J. V. Ortiz, Quantum-Mechanical Prediction of Thermochemical Data, Kluwer, 2001

26 J. Ortiz, J. Chem. Phys., 1996, 104, 7599-7605. 
27 M. J. Frisch, G. W. Trucks, H. B. Schlegel, G. E. Scuseria, M. A Robb, J. R. Cheeseman, G. Scalmani, V. Barone, B. Mennucci, G. A. Petersson, H. Nakatsuji, M. Caricato, X. Li, H. P. Hratchian, A. F. Izmaylov, J. Bloino, G. Zheng, J. L. Sonnenberg, M. Hada, M. Ehara, K. Toyota, R. Fukuda, J. Hasegawa, M. Ishida, T. Nakajima, Y. Honda, O. Kitao, H. Nakai, T. Vreven, J. A Montgomery, Jr., J. E. Peralta, F. Ogliaro, M. Bearpark, J. J Heyd, E. Brothers, K. N. Kudin, V. N. Staroverov, R. Kobayashi, J. Normand, K. Raghavachari, A. Rendell, J. C. Burant, S. S. Iyengar, J. Tomasi, M. Cossi, N. Rega, J. M. Millam, M. Klene, J. E. Knox, J. B. Cross, V. Bakken, C. Adamo, J. Jaramillo, R. Gomperts, R. E. Stratmann, O. Yazyev, A. J. Austin, R. Cammi, C. Pomelli, J. W. Ochterski, R. L. Martin, K. Morokuma, V. G. Zakrzewski, G. A. Voth, P. Salvador, J. J. Dannenberg, S. Dapprich, A. D. Daniels, Ö. Farkas, J. B. Foresman, J. V. Ortiz, J. Cioslowski and D. J. Fox, Gaussian 09 Revision B.1, Gaussian Inc, Wallingford CT, 2009.

28 T. Crawford, H. Schaefer and T. Lee, J. Chem. Phys., 1996, 105, 1060-1069.

29 T. Lee, A. Rendell, K. Dyall and D. Jayatilaka, J. Chem. Phys., 1994, 100, 7400-7409.

30 M. Schmidt, K. Baldridge, J. Boatz, S. Elbert, M. Gordon, J. Jensen, S. Koseki, N. Matsunaga, K. Nguyen, S. Su,
T. Windus, M. Dupuis and J. Montgomery, J. Comput. Chem., 1993, 14, 1347-1363.

31 M. S. Gordon and M. Schmidt, in Advances in electronic structure theory: GAMESS a decade later, ed. C. E. Dykstra, G. Frenking, K. S. Kim and G. E. Scuseria, Elsevier, Amsterdam, 2005, pp. 1167-1189.

32 V. Mozhayskiy and A. Krylov, ezSpectrum, http://iopenshell.usc. edu/downloads.

33 L. Koziol, V. Mozhayskiy, B. Braams, J. Bowman and A. Krylov, J. Phys. Chem. A, 2009, 113, 7802-7809.

34 F. Duschinsky, Acta Physicochim. URSS, 1937, 7, 551-566.

35 U. Fano and J. Cooper, Rev. Mod. Phys., 1968, 40, 441-507.

36 T. Olney, N. Cann, G. Cooper and C. Brion, Chem. Phys., 1997, 223, 59-98.

37 W. B. DeMore, S. P. Sander, D. M. Golden, R. F. Hampson, M. J. Kurylo, C. J. Howard, A. R. Ravishankara, C. E. Kolb and M. J. Molina, Chemical Kinetics and Photochemical Data for Use in Stratospheric Modeling, Evaluation Number 12, January 15, 1997.

38 P. Limao-Vieira, S. Eden, P. Kendall, N. Mason and S. Hoffmann, Chem. Phys. Lett., 2002, 364, 535-541.

39 K. Gill and R. Hites, J. Phys. Chem. A, 2002, 106, 2538-2544.

40 S. Corchnoy and R. Atkinson, Environ. Sci. Technol., 1990, 24, $1497-1502$. 